\title{
Caracterização farmacognóstica, fitoquímica e avaliação in silico da atividade de monoterpenos isolados da espécie Dysphania ambrosioides (L.) Mosyakin \& Clemants
}

\author{
Pharmacognostic, phytochemical characterization and in silico evaluation of the activity of \\ monoterpens isolated from the species Dysphania ambrosioides (L.) Mosyakin \& Clemants \\ Caracterización farmacognóstica, fitoquímica y evaluación sílica de la actividad de monoterpenos \\ aislados de la especie Dysphania ambrosioides (L.) Mosyakin \& Clemants
}

Recebido: 01/06/2021 | Revisado: 13/06/2021 | Aceito: 22/06/2021 | Publicado: 05/07/2021

\author{
Ana Carolina Sousa Quaresma \\ ORCID: https://orcid.org/0000-0001-9336-8725 \\ Universidade Federal do Pará, Brasil \\ E-mail: carolinquaresma@gmail.com \\ Paulo Ricardo de Souza Melo \\ ORCID: https://orcid.org/0000-0001-7258-9203 \\ Universidade Federal do Pará, Brasil \\ E-mail: paulo.ricardof50@gmail.com \\ Dayse Lúcia do Nascimento Brandão \\ ORCID: https://orcid.org/0000-0003-0633-6069 \\ Universidade Federal do Pará, Brasil \\ E-mail: daysena_25@yahoo.com.br \\ Maria Fâni Dolabela \\ ORCID: https://orcid.org/0000-0003-0804-5804 \\ Universidade Federal do Pará, Brasil \\ E-mail: fanidolabela20@gmail.com
}

\begin{abstract}
Resumo
O estudo tem por objetivo descrever os resultados obtidos dos parâmetros de qualidade farmacognóstico, prospecção fitoquímica e estudos in silico de monoterpenos isolados de Dysphania ambrosioides. Utilizou-se folhas e caules que foram secas e pulverizadas, os pós foram submetidos a testes farmacognóstico. Por meio de maceração com etanol obteve-se os extratos de caule (EEC) e de folhas (EEF), seguido de prospecção fitoquímica através de cromatografia em camada delgada. Para os estudos in silico selecionou-se os terpenos (1S,2S,3R,4S)-1-metil-4-(propan-2-il)ciclohexano-1,2,3,4-tetrol (A); 1,2,3,4-tetrahidroxi-p-metano (B); (1R,2S)-3-pmeten-1,2-diol (C); (1R,4S)-p-met-2-

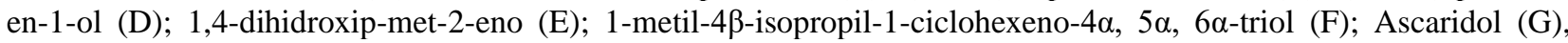
onde utilizou-se os programas Marvin JS, PreADMET, PASS online, Molinspiration Online, para avaliação dos aspectos físico-químicos, farmacocinéticos, toxicológicos e predição de atividade. Os pós foram classificados como grossos, com teor de umidade dentro dos parâmetros recomendados, sendo ausente as saponinas, pH neutro e baixa densidade. Na prospecção fitoquímica foram detectados triterpenos e esteroides, saponinas e heterosídeos flavônicos. Todos os compostos seguem a regra de Lipinski; foram bem absorvidos no trato gastrointestinal; C, D, E e G ligam-se fortemente às proteínas plasmáticas; $\mathrm{C}$ e D distribuíram amplamente no sistema nervoso central; $\mathrm{D}$ e $\mathrm{E}$ não foram metabolizadas pelo CYP e todas inibiram CYP; A e B não foram mutagênicas e F e G são carcinogênicas. As drogas vegetais apresentaram boa qualidade, sendo os terpenos presentes nestas amostras. Os estudos in silico apontam que as moléculas as moléculas C, F e G são promissoras para atividade anti-inflamatória, antineoplásica e antiprotozoária. Palavras-chave: Mastruz; Terpeno; Estudo in sílico.
\end{abstract}

\begin{abstract}
The study aims to describe the results obtained from pharmacognostic quality parameters, phytochemical prospection and in silico studies of monoterpenes isolated from Dysphania ambrosioides. Leaves and stems were used, which were dried and sprayed, the powders were submitted to pharmacognostic tests. Stem (EEC) and leaf (EEF) extracts were obtained through maceration with ethanol, followed by phytochemical prospecting through thin layer chromatography. For in silico studies the terpenes were selected: (1S,2S,3R,4S)-1-methyl-4-(propan-2-yl)cyclohexane-1,2,3,4-tetrol (A); 1,2,3,4-tetrahydroxy-p-methane (B); (1R,2S)-3-pmethen-1,2-diol (C); (1R,4S)-p-met2-en-1-ol (D); 1,4-dihydroxyp-met-2-ene (E); 1-methyl-4 $\beta$-isopropyl-1-cyclohexene-4 $\alpha, 5 \alpha, 6 \alpha$-triol (F); Ascaridol (G), where the programs Marvin JS, PreADMET, PASS online, Molinspiration Online were used, to evaluate the physical-chemical, pharmacokinetic, toxicological and activity prediction aspects. The powders were classified as coarse, with moisture content within the recommended parameters, saponins, neutral $\mathrm{pH}$ and low density being absent. In the phytochemical prospection, triterpenes and steroids, saponins and flavonic heterosides were detected. All
\end{abstract}


compounds follow Lipinski's rule; were well absorbed from the gastrointestinal tract; C, D, E and G are strongly bound to plasma proteins; $\mathrm{C}$ and $\mathrm{D}$ distributed widely in the central nervous system; D and $\mathrm{E}$ were not metabolized by CYP and all inhibited CYP; A and B were not mutagenic and F and G are carcinogenic. The plant drugs showed good quality, and the terpenes were present in these samples. In silico studies indicate that molecules $\mathrm{C}, \mathrm{F}$ and $\mathrm{G}$ are promising for anti-inflammatory, antineoplastic and antiprotozoal activity.

Keywords: Mastruz; Terpene; In silico study.

\begin{abstract}
Resumen
El estudio tiene como objetivo describir los resultados obtenidos a partir de parámetros de calidad farmacognósticos, prospección fitoquímica y estudios in silico de monoterpenos aislados de Dysphania ambrosioides. Se utilizaron hojas y tallos, que se secaron y pulverizaron, los polvos se sometieron a pruebas farmacognósticas. Los extractos de tallo (EEC) y hoja (EEF) se obtuvieron mediante maceración con etanol, seguida de prospección fitoquímica mediante cromatografía en capa fina. Para estudios in silico se seleccionaron los terpenos (1S, 2S, 3R, 4S) -1-metil-4- (propan2-il)-ciclohexano-1,2,3,4-tetrol (A); 1,2,3,4-tetrahidroxi-p-metano (B); (1R, 2S) -3-pmeten-1,2-diol (C); (1R, 4S) -p-

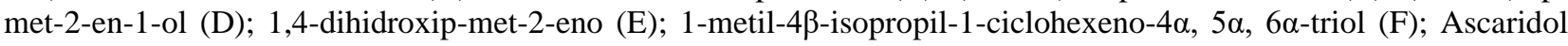
(G), donde se utilizaron los programas Marvin JS, PreADMET, PASS online, Molinspiration Online, para evaluar los aspectos físico-químicos, farmacocinéticos, toxicológicos y de predicción de actividad. Los polvos se clasificaron como gruesos, con contenido de humedad dentro de los parámetros recomendados, sin saponinas, pH neutro y baja densidad. En la prospección fitoquímica se detectaron triterpenos y esteroides, saponinas y heterósidos flavónicos. Todos los compuestos siguen la regla de Lipinski; se absorbieron bien en el tracto gastrointestinal; C, D, E y G se unen fuertemente a las proteínas plasmáticas; $C$ y D distribuidos ampliamente en el sistema nervioso central; D y E no fueron metabolizados por CYP y todos inhibieron CYP; A y B no fueron mutagénicos y $\mathrm{F}$ y $\mathrm{G}$ son cancerígenos. Los medicamentos vegetales mostraron buena calidad y los terpenos estaban presentes en estas muestras. Los estudios in silico indican que las moléculas $\mathrm{C}, \mathrm{F}$ y $\mathrm{G}$ son prometedoras para la actividad antiinflamatoria, antineoplásica y antiprotozoaria.
\end{abstract}

Palabras clave: Mastruz; Terpeno; Estudio in silico.

\title{
1. Introdução
}

A espécie Dysphania ambrosioides (L.) Mosyakin \& Clemants, com sinonímia Chenopodium ambrosioides L., da família Amaranthaceae (Mosyakin e Clemants, 2008), é uma planta nativa da América Central e do Sul, com distribuição cosmopolita, ocorrendo principalmente em regiões de clima tropical (Lorenzi \& Matos, 2002; Monzote, et al., 2009).

Popularmente é conhecida como erva-de-santa-maria, mastruz ou mastruço, sendo amplamente utilizada na medicina tradicional na forma de chás, batidas, xaropes ou infusões para tratar afecções como: verminoses, gripe, tosse, pneumonia, inflamação uterina (Moraes, et al., 2005; Oliveira, et al., 2010; Barbosa, et al., 2011), infecções parasitárias (Calzada, et al., 2010), alívio das dores musculares (Garcia, Domingues \& Rodrigues, 2010) e inflamações em geral (Cartaxo, et al., 2010; Ramos, et al., 2011).

A partir dos relatos etnofarmacológicos, estudos puderam elucidar a atividade anti-inflamatória de extrato aquoso desta espécie foi demonstrada através de testes in vitro e in vivo (Trindade, et al., 2021), bem como a atividade antinociceptiva através dos testes de contorção induzida por ácido acético e placa quente, que sugeriram a resposta do o extrato aquoso das folhas na inibição dos mecanismos de dor periférica e central (Hallal, et al., 2010). Além disso, o extrato aquoso obtido das folhas demonstrou potencial antitumoral em linhagem celular de leucemia (Pandiangan, et al., 2020). Outro estudo avaliou a atividade leishmanicida do extrato hidroalcoólico das folhas, sendo observado a diminuição no índice de infecção de macrófagos por Leishmania amazonenses, a partir do aumento da produção de óxido nítrico (Lima Júnior, et al., 2014).

A atividade contra epimastigota de Tripanossoma cruzi de terpenos isolados de D. ambrosioides foi avaliada, observando que o terpeno ascaridol se mostrou mais promissor (Kiuchi, et al., 2002). Em relação à atividade antioxidante da espécie, estudos sugerem que os flavonoides e glicosídeos são responsáveis por essa atividade (Arisawa, et al., 1971; Jain, et al., 1990).

Considerada a relação de determinadas classes de metabólitos com a atividade biológica, estudos fitoquímicos desta espécie demostraram que já foram isolados os seguintes compostos terpênicos: ascaridol, 1,2,3,4-tretahidroxi-p-metano (Grassi, 2011), (1S,2S,3R,4S)-1-metil-4-(propan-2-il)-ciclohexano-1,2,3,4-tetrol, (1R,2S)-3-p-meten-1,2-diol, (1R,4S)-p-met- 


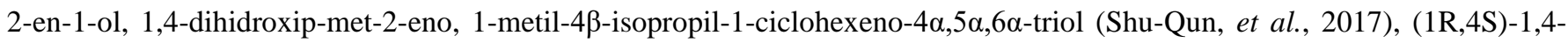
dihidroxi-p-ment-2-eno e (1R,2S,3S,4S)-1,2,3,4-tetrahidroxi-p-mentano (Ahmed, 2000). Além disso, foram isolados os polifenóis chenopodiumamina A, chenopodiumamina B, chenopodiumamina C, chenopodiumamina D e chenopodiumoside A (Kun, et al., 2015), as cumarinas escopoletina e 1,2-benzopirona (Shan \& Khan, 2017) os e flavonoides kaempferol, isoramnetina, ambroside (Arisawa, et al., 1971; Jain, et al., 1990), kaempferol-3-O- $\alpha$-L-1C4-rhamnosil-(1"' $\rightarrow 2$ '”)- $\beta$-D-4C1xilopiranospideo e kaempferol-7-O- $\alpha$-L-1C4-ramnopiranosídeo (Ghareeb, et al., 2016).

Nota-se que apesar de promissora para várias atividades biológicas, ainda há carência de estudos que relacionem seus metabólitos aos aspectos farmacocinéticos, toxicológicos e físico-químicos. O presente estudo avaliou os parâmetros farmacognósticos da droga vegetal de D. ambrosioides, bem como realizou prospecção fitoquímica dos seus extratos seguida de seleção de moléculas já isoladas da espécie e submissão destas a estudo in silico visando avaliar aspectos farmacocinéticos, toxicológicos, físico-químicos e predição de atividades biológicas.

\section{Metodologia}

\subsection{Coleta e processamento do material vegetal}

Neste estudo foram utilizados folhas e caules de Dysphania ambrosioides, que foram coletados no município de Santa Izabel-PA. O material vegetal foi identificado por um especialista e teve sua exsicata depositada no Herbário da Embrapa Amazônia Oriental (IAN) sob o número de registro 200572. Posteriormente, o material vegetal foi lavado e seco em estufa de ar circulante a $37-40^{\circ} \mathrm{C}$ por 7 dias. Após seco, o material foi separado em folhas e caules, e foram pulverizados em moinho de facas para obtenção dos pós da folha e do caule.

\subsection{Estudos Farmacognósticos}

Os pós obtidos das folhas e dos caules de D. ambrosioides foram submetidos a estudos de caracterização farmacognóstica, onde realizou-se a determinação dos parâmetros de densidade (Lachman, Lieberman \& Kanig, 2001), granulometria, pH, perda por dessecação e índice de espuma (Brasil, 2019). Todos os parâmetros analisados foram realizados em triplicata.

\subsection{Estudos fitoquímicos}

Para obtenção dos extratos, os pós da folha e do caule foram submetidos separadamente à maceração exaustiva em proporção adequada de material para etanol (1:10). Após esse período, a solução extrativa foi filtrada e concentrada em evaporador rotativo sob pressão reduzida, obtendo-se o extrato etanólico da folha (EEF) e o extrato etanólico do galho (EEC).

Os extratos foram submetidos a prospecção fitoquímica através de cromatografia de camada delgada (CCD). Foram pesquisadas as seguintes classes de metabólitos: triterpenos e esteroides, geninas flavônicas, heterosídeos flavônicos, cumarinas, saponinas, polifenóis, alcaloides, taninos e heterosídeos antracênicos. Para cada metabólito utilizou-se uma fase móvel e revelador específico (Tabela 1) (Wagner, et al., 1984). 
Tabela 1. Condições utilizadas nas avaliações em cromatografia em camada delgada.

\begin{tabular}{|c|c|c|}
\hline Metabólito & Fase móvel & Revelador \\
\hline Alcaloides & $\begin{array}{l}\text { Clorofórmio: Metanol: Hidróxido de } \\
\text { Amônio }(85: 15: 0,2) .\end{array}$ & Dragendorff. \\
\hline Cumarinas & $\begin{array}{c}\text { Tolueno: Éter (1:1) saturado com Ácido } \\
\text { acético (5 gotas). }\end{array}$ & Hidróxido de potássio a 5\% em metanol. \\
\hline Geninas Flavônicas & $\begin{array}{l}\text { Clorofórmio: Acetato de etila: Ácido } \\
\text { fórmico }(60: 40: 2,5) \text {. }\end{array}$ & Cloreto de alumínio a $2 \%$ em metanol. \\
\hline Polifenóis & $\begin{array}{c}\text { Acetato de etila: Ácido Fórmico: Ácido } \\
\text { acético: Água (100:11:11:27). }\end{array}$ & $\begin{array}{c}\text { Soluções de ferricianeto de potássio a } 1 \% \text { e } \\
\text { cloreto férrico a } 2 \% .\end{array}$ \\
\hline Saponinas & Clorofórmio: Metanol (95:5). & Anisaldeído. \\
\hline Triterpenos e Esteróides & Hexano: Acetato de etila $(8: 2)$ & Reagente de Lieberbanm-Burchard \\
\hline Heterosídeos flavônicos & $\begin{array}{l}\text { Acetato de etila: Ácido fórmico: Ácido } \\
\text { acético: Água (100:11:11:27) }\end{array}$ & Cloreto de alumínio a $2 \%$ em metanol. \\
\hline Taninos & $\begin{array}{l}\text { Acetato de etila: Ácido fórmico: Ácido } \\
\text { acético: Água (100:11:11:27) }\end{array}$ & $\begin{array}{l}\text { Soluções ferrocianeto de potássio a } 1 \% \text { e } \\
\text { cloreto férrico a } 2 \% \text {. }\end{array}$ \\
\hline $\begin{array}{l}\text { Heterosídeos } \\
\text { antracênicos }\end{array}$ & $\begin{array}{l}\text { Acetato de etila: Metanol: Água } \\
(81: 11: 8)\end{array}$ & Hidróxido de potássio a $5 \%$ em metanol. \\
\hline
\end{tabular}

Fonte: Brandão (2012), adaptado de Wagner, et al. (1984).

\subsection{Seleção de moléculas}

Baseado no resultado da prospecção fitoquímica, selecionou-se os terpenos detectados em ambas as amostras de folhas e caules (EEC e EEF) e com isso, realizou-se um levantamento de estudos que já tenham isolados moléculas pertencentes a classe, sendo assim, selecionou-se da espécie as principais moléculas de monoterpenos isolados com possíveis atividades biológicas.

\subsection{Estudos in silico}

O screening virtual é uma técnica simples e amplamente utilizada que se baseia na busca por similaridade em que uma conhecida estrutura-alvo bioativa é procurada em uma base de dados para identificar as moléculas mais similares, uma vez que estas serão as mais prováveis de terem atividade de interesse, o que naturalmente as torna candidatas para testes biológicos.

Para este estudo foram utilizados os programas Marvin Js para desenhar as estruturas químicas; ADMET Prediction absorção, distribuição, metabolização, excreção e toxicidade (versão 2.0 Copyright $^{\oplus}$ 2005; Preadmet, 2021) para as predições farmacocinéticas e toxicológicas; e PASS online Prediction of activity spectra for substances (way2drug.com ${ }^{\oplus} 2011$ versão 2.0 - 2021; Pass online, 2021) para as previsões de espectro de atividades biológicas e o servidor Molinspiration Online Property Calculator Tookilt (www.molinspiration.com; Molinspiration, 2021) para avaliar as características físico-químicas dos compostos.

Os dados de ADMETox foram comparados com substâncias semelhantes a partir da regra de Lipinski ou "regra dos cinco", em que se estabelece que uma molécula deve apresentar valores para quatro parâmetros múltiplos de cinco: $\log \mathrm{P} \leq 5$, massa molecular $\leq 500 \mathrm{Da}$, aceptores de ligação de hidrogênio $\leq 10$ e doadores de ligação de hidrogênio $\leq 5$ (Lipinski, 2004).

Para estudos farmacocinéticos avaliou-se a absorção intestinal (human intestinal absorption = HIA) considerando os seguintes parâmetros: 0-20\% (baixa absorção), 20-70\% (moderada absorção) > 70\% (alta absorção) (Yee, 1997) e a permeabilidade em células Caco 2 e MDCK: alta permeabilidade $>70 \mathrm{~nm} / \mathrm{sec}$, média permeabilidade $4-70 \mathrm{~nm} / \mathrm{sec}$ e baixa permeabilidade < $4 \mathrm{~nm} / \mathrm{sec}$ (Yazdanian, et al., 1998). Para a distribuição das substâncias utilizou os seguintes referenciais: ligado fortemente à albumina > 90\% e ligação de moderada a fraca à albumina < 90\% (Preadmet, 2021). Os critérios selecionados para o quanto a molécula consegue perpassar a barreira hematoencefálica (BHE) foram: atravessa livremente a BHE > 2,0, atravessa de forma moderada 2,0-0,1 e atravessa de forma fraca ou não atravessa < 0,1 (Ajay, et al., 1999). 
Para avaliar os resultados relacionados ao metabolismo dos constituintes utilizou-se: se as substâncias sofrem metabolismo de fase 1, se inibem alguma CYP e quais CYP foram inibidas. Substâncias que inibem duas ou mais CYP, especialmente CYP3A4 e CYP2C9, podem interferir no metabolismo de uma grande quantidade de fármacos e outras substâncias, pode contribuir para a elevação da toxicidade. Para substâncias que inibem uma única CYP, pode ocorrer a redução da quantidade de fármacos que induzem interação farmacocinética com a molécula testada. Substâncias não inibidoras e não indutoras de CYP são consideradas substâncias ideais, por não interferir no metabolismo de outros fármacos.

Para a predição de mutagenicidade utilizou-se do teste de Ames, em que estirpes de Salmonella typhimurium (TA98, TA100, TA1525) com alteração do gene da histidina foram testadas. A variável a ser testada é a capacidade do agente mutagênico provocar reversão para o crescimento em meio sem histidina (Ames et al., 1875). Para a interpretação dos resultados deste teste considerou-se positivo quando houver reversão da mutação em uma ou mais bactérias em meio sem histidina e negativo quando não se observar reversão da mutação nas mesmas. Para falso positivo, ou seja, não houver reversão da mutação em nenhum clone da bactéria e mesmo assim o programa classificou mutagênico, o resultado foi desconsiderado.

Em relação a avaliação dos critérios toxicidade de organismos marinhos, considerou-se os seguintes parâmetros: toxicidade em algas: tóxico < 1mg/L e não tóxicos > $1 \mathrm{mg} / \mathrm{L}$ (Costa, et al., 2008); toxicidade em Daphnia sp.: tóxico < 0,22 $\mu \mathrm{g} / \mathrm{mL}$ e não tóxico > 0,22 $\mu \mathrm{g} / \mathrm{mL}$ (Guilhermino, et al., 2000); toxicidade em peixes Medaka e Minnow: muito tóxico < 1mg/L, tóxico entre 1-10 mg/L, prejudiciais 10-100 mg/L e não tóxico > $100 \mathrm{mg} / \mathrm{L}$ (Zucker, 1985). Para a predição de carcinogenicidade dos compostos em roedores (Rodent Carcinogenicity) foi realizado a partir dos dados da National Toxicology Program (NTP) e FDA (Food and Drug Administration). Os resultados foram expressos em carcinogênico (+) e não carcinogênico (-).

A atividade biológica das substâncias testadas foi avaliada por similaridade com mais de 250.000 substâncias biologicamente ativas, incluindo fármacos e compostos tóxicos. Como critério de avaliação adotou-se Pa (Probabilidade de atividade) de 0,7, ou seja, 70\% (Filimonov, et al., 1995).

\section{Resultados e Discussão}

As análises de granulometria demonstram que os pós da folha e do caule de D. ambrosioides, podem ser classificados como um pó grosso (Tabela 2). Pós grossos diminuem a superfície de contato do material vegetal com líquido extrator podendo interferir na eficiência do processo extrativo, diminuindo rendimento da extração.

Tabela 2. Análise de granulometria do pó da folha e do caule de D. ambrosioides.

\begin{tabular}{ccc}
\hline Abertura nominal da & \multicolumn{2}{c}{ Média (\%) $\mathbf{\text { desvio padrão }}$} \\
\cline { 2 - 3 } malha & Folha & Caule \\
\hline $1,70 \mathrm{~mm}$ & $3,1 \pm 0,04$ & $68,3 \pm 0,88$ \\
$710 \mu \mathrm{m}$ & $26,5 \pm 0,44$ & $21,9 \pm 0,89$ \\
$355 \mu \mathrm{m}$ & $40 \pm 0,51$ & $4,9 \pm 0,46$ \\
$180 \mu \mathrm{m}$ & $23,2 \pm 0,76$ & $2,4 \pm 0,25$ \\
$125 \mu \mathrm{m}$ & $4,1 \pm 0,35$ & $0,9 \pm 0,11$ \\
Coletor & $3 \pm 0,49$ & $1,2 \pm 0,03$ \\
\hline & Fonte: Autores.
\end{tabular}

Outro parâmetro avaliado foi à densidade aparente, onde a densidade dos pós das folhas $(0,23 \mathrm{~g} / \mathrm{mL} \pm 0,14)$ e dos caules $(0,57 \mathrm{~g} / \mathrm{mL} \pm 0,23)$, foram consideradas baixas (Tabela 3$)$, se comparada com a água $\left(1,0 \mathrm{~g} / \mathrm{cm}^{3}\right)$ que é o padrão para sólidos e líquidos, ocupando um volume maior (Montanheiro, 1990). Este parâmetro expressa a relação entre a massa de material seco e o volume real ocupado por essas partículas, é importante por influenciar diretamente no preparo de extratos, 
pois o pó de baixa densidade necessita de uma maior quantidade de solvente durante o processo de extração para evitar a flutuação das partículas (Amarante, 2010; Zorzeto, et al., 2014).

$\mathrm{Na}$ determinação do $\mathrm{pH}$, verificou-se que o $\mathrm{pH}$ obtido do pó da folha e do caule foi de 7,31 e 7,13, respectivamente (Tabela 3). Sugerindo que os pós desta planta contêm compostos de caráter alcalino ou fracamente ácidos. O índice de espuma de soluções dos pós analisados apresentara altura da espuma em todos os tubos inferior a $1 \mathrm{~cm}$, com um índice de espuma menor que 100 (Tabela 3), sugerindo a ausência de saponinas. Outros estudos descrevem ausência de saponinas em solução de pós das folhas e de partes aéreas de D. ambrosioides (Valério, 2014; Fontenele, 2017). Além disso, não foram encontrados estudos que descrevem o isolamento de saponinas desta espécie.

Em relação a análise de perda por dessecação, observou-se que os pós da folha e caule de D. ambrosioides, apresentaram percentual de 11,6\% e 11,7\%, respectivamente (Tabela 3), esses valores encontram-se dentro dos limites estabelecidos pela Farmacopeia Brasileira (8-12\%). O que indica que o material vegetal passou por secagem e armazenamento eficientes, e sugere uma boa estabilidade microbiológica e química (Brasil, 2019; Simões, et al., 2010).

Tabela 3. Análise farmacognóstica do pó da folha e do caule de D. ambrosioides.

\begin{tabular}{ccc}
\hline PARÂMETRO & MÉDIA \pm DESVIO PADRÃO & MÉDIA \pm DESVIO PADRÃO \\
\hline Perda por dessecação $(\%)$ & $11,6 \pm 0,92$ & $11,7 \pm 0,80$ \\
pH & $7,31 \pm 0,03$ & $7,13 \pm 0,07$ \\
Índice de espuma & $<100$ & $<100$ \\
Densidade do pó $(\mathrm{g} / \mathrm{mL})$ & $0,23 \pm 0,14$ & $0,57 \pm 0,23$ \\
\hline
\end{tabular}

Fonte: Autores.

Os rendimentos dos extratos etanólicos foram de 8,73\% para a folha e 5,35\% para o caule. Sendo considerados satisfatórios se comparado a um etudo anterior, que utilizou método de extração similar, e obteve-se um percentual de rendimento de 2,18\% das folhas e 1,97\% para os caules (Grassi, 2011).

A abordagem fitoquímica dos extratos etanólicos obtido das folhas (EEF) e dos caules (EEC) de D. ambrosioides revelou bandas sugestivas para presença de saponinas (Tabela 4), que apesar do resultado negativo no teste de determinação de índice de espuma, o método de CCD a detectou, indicando a diferença de sensibilidade dos métodos; triterpenos e esteroides, em que na literatura, podem ser encontrados compostos já isolados desta classe a partir do extrato alcoólico (Shu-Qun, et al., 2017) e heterosídeos flavônicos, os quais ainda não foi isolado nenhum composto.

Na prospecção fitoquímica do extrato etanólico do caule detectou-se a presença de alcaloides e cumarinas (Tabela 4), desta espécie já foram isoladas as cumarinas escopoletina e 1,2-benzopirona e o alcaloide 1-piperoilpiperidina (Shah \& Khan, 2017), entretanto, somente estudos de identificação podem comprovar a presença destes metabólitos. Nota-se as diferentes classes de metabólitos já isoladas da espécie. Além disso, estudos revelam que a planta possui valores consideráveis de terpenos, sendo estes de importância biológica e facilmente isolados (Dembitsky, 2008).

A partir da análise fitoquímica de extratos da folha e do caule, onde observou-se a presença de terpenos (Tabela 4), e ao levantamento bibliográfico que sugerem as diversas atividades biológicas promissoras para a classe (Shu-Qun, et al., 2017; Grassi, 2011; Ahmed, 2000). Selecionou-se sete monoterpenos isolados da espécie (Figura 1) e realizou-se estudos in silico dos aspectos farmacocinéticos, toxicológicos, físico-químicos e de atividade biológica dos compostos terpênicos isolados da $D$. ambrosioides. 
Tabela 4 - Prospecção fitoquímica dos extratos etanólicos de folha e caule de Dysphania ambrosioides.

\begin{tabular}{ccc}
\hline METABÓLITOS & \multicolumn{2}{c}{ AMOSTRAS } \\
\cline { 2 - 4 } SECUNDÁRIOS & EEF & EEC \\
\hline Alcaloides & - & + \\
Cumarinas & - & + \\
Taninos & - & - \\
Geninas flavônicas & - & + \\
Heterosídeos flavônicos & + & - \\
Polifenóis & - & + \\
Saponinas & + & + \\
\hline
\end{tabular}

Legenda: (+) presença; (-) ausência; EEF - extrato etanólico da folha; EEC - extrato etanólico do caule. Fonte: Autores.

Figura 1. Monoterpenos isolados de D. ambrosioides.

(A)

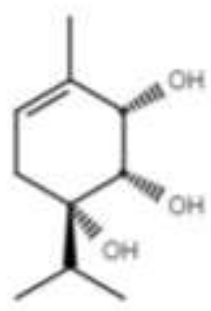

(B)

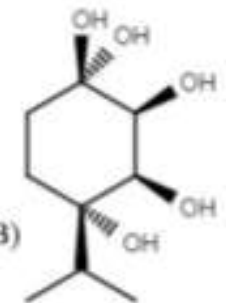

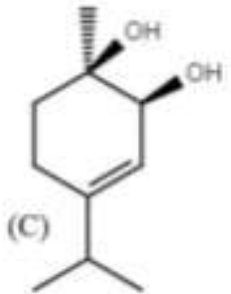

(D)

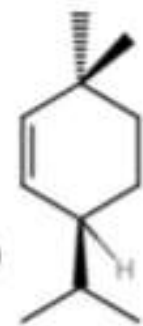<smiles>CC(C)[C@]1(O)C=CC(C)(C)CC1</smiles><smiles>CC1=CC[C@@](O)(C(C)C)[C@H](O)[C@@H]1O</smiles>

(G)

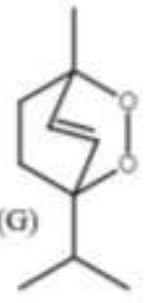

Legenda: (A) (1S,2S,3R,4S)-1-metil-4-(propan-2-il)ciclohexano-1,2,3,4-tetrol; (B) 1,2,3,4-tetrahidroxi- p-metano; (C) (1R,2S)-3-p-meten-

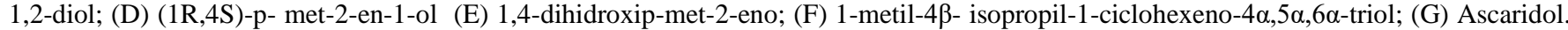
Fonte: Autores.

$\mathrm{Na}$ Tabela 5, as moléculas A e B apresentaram $\log \mathrm{P}$ de 0,23 , TPSA no valor de 80,91, número de aceptores e doadores de hidrogênio igual a 4 e massa molar de 204,27; a molécula C apresentou logP de 1,45, TPSA de 40,46, valores de aceptores e doadores de hidrogênio igual a 2 e massa molar no valor de 170,25; as moléculas D e E obtiveram logP de 2,80, TPSA 20,23, número de aceptores e doadores de hidrogênio igual a 1 e massa molar de 154,25; A molécula F apresentou logP de 0,77, TPSA de 60,68, número de doadores e aceptores de hidrogênio igual a 3 e massa molar de 186,25; por fim, a molécula G mostrou um $\log \mathrm{P}$ de 2,65, TPSA de 18,47, uma quantidade de 2 aceptores de hidrogênio e nenhum doador e massa molar de 168,24; todas as moléculas apresentaram apenas uma ligação rotacionável.

A partir desses resultados, nota-se que todas as moléculas contemplam de forma eficiente a regra dos cinco de Lipinski (Lipinski, 2005). Além disso, parâmetros como estes influenciam diretamente na resposta farmacocinética de cada substância, uma vez que, fármacos ideais devem apresentar polaridade média, observou-se que os monoterpenos A, B e F apresentam um $\log \mathrm{P}$ baixo, indicando que possivelmente são compostos mais hidrossolúveis e polares, desta forma tem moderada absorção intestinal em relação às outras moléculas do estudo que apresentam $\log \mathrm{P}$ na faixa de 1 a 3 expressando parâmetros farmacocinéticas ideais (Tabela 5; Kubinyi, 1977). Essas mesmas moléculas (A, B e F) indicam maior dificuldade 
de ligação com possível receptor por apresentarem um valor de TPSA maior que $60 \AA$ (80,91; 80,91 e 60,68, respectivamente), o TPSA está relacionado à formação de ligações de hidrogênio e à polaridade, influenciando na interação da molécula com a superfície polar das membranas e a sua migração para a porção lipídica, ou seja, quanto mais o valor se aproxima de $140 \AA$, mais difícil é essa interação (Tabela 5; Veber, et al., 2002).

Quanto a quantidade de grupos aceptores de hidrogênio, as moléculas C, D, E e G apresentam valores abaixo de 10 e quantidade de doadores de hidrogênio menor que 5 (Tabela 5), e as moléculas A, B e F quantidades mais próximas da referência de doadores de hidrogênio (4, 4 e 3, respectivamente; Tabela 5) o que pode prejudicar a permeabilidade destas através de membranas biológicas (Lipinski, et al., 1997). Quanto ao número de ligações rotacionáveis, todas as moléculas apresentaram a quantidade de uma única ligação rotacionável (Tabela 5), indicando que a conformação destas moléculas não irá comprometer sua biodisponibilidade, uma vez que um menor número de ligações como estas é ideal para garantir a estabilidade conformacional das moléculas no meio (Veber, et al., 2002).

Em relação a massa molar (Tabela 5) observou-se que as moléculas A, B e F tiveram maior massa, o que associado ao $\log \mathrm{P}$ reforça a propriedade hidrossolúvel destas moléculas, visto que o aumento do peso molecular pode dificultar a concentração desses compostos na superfície do epitélio intestinal e, por consequência, reduzir a absorção destes (Di e Kerns, 2015). As moléculas C, D, E e G (Tabela 5) apresentam maior $\log \mathrm{P}(1,45 ; 2,80 ; 2,80$ e 2,65, respectivamente) e menor massa molar $(170,25 ; 154,25 ; 154,25$ e 168,24 , respectivamente) sugerindo que possivelmente estas moléculas têm maior lipossolubilidade e assim maior facilidade em perpassar as barreiras.

Tabela 5. Propriedades físico-químicas de monoterpenos isolados de D. ambrosioides.

\begin{tabular}{ccccccc}
\hline Monoterpenos & LogP & TPSA & nALH & nDLH & nROTB & MM \\
\hline A & 0,23 & 80,91 & 4 & 4 & 1 & 204,27 \\
B & 0,23 & 80,91 & 4 & 4 & 1 & 204,27 \\
C & 1,45 & 40,46 & 2 & 2 & 1 & 170,25 \\
D & 2,80 & 20,23 & 1 & 1 & 1 & 154,25 \\
E & 2,80 & 20,23 & 1 & 1 & 1 & 154,25 \\
F & 0,77 & 60,68 & 3 & 3 & 1 & 186,25 \\
G & 2,65 & 18,47 & 2 & 0 & 1 & 168,24 \\
Referência & $\leq 5$ & $\leq 140 \AA$ & $\leq 10$ & $\leq 5$ & $\leq 10$ & $\leq 500 \mathrm{Da}$ \\
\hline
\end{tabular}

Legenda: Regra de Lipinski: LogP - coeficiente de partição óleo-água; TPSA - área de superfície polarizada topológica; nALH - aceptores de ligação de hidrogênio; nDLH - número de grupos de doadores de hidrogênio; nROTB - número de ligações rotacionáveis; MM - massa molar, Fonte: Autores.

Para os parâmetros de absorção (Tabela 5), as moléculas A e B apresentaram valores de $2 \mathrm{~nm} / \mathrm{sec}$ para absorção em MDCK, $14 \mathrm{~nm} / \mathrm{sec}$ em Caco-2 e 58\% de absorção intestinal humana; já a molécula C apresentou os valores de $373 \mathrm{~nm} / \mathrm{sec}$ para MDCK e $18 \mathrm{~nm} / \mathrm{sec}$ para células Caco-2 e percentual de absorção de 88\% em intestino humano; a molécula D mostrou 188 $\mathrm{nm} / \mathrm{sec}$ em células MDCK, $50 \mathrm{~nm} / \mathrm{sec}$ em células Caco-2 e 100\% de absorção intestinal humana; a molécula E valores de 0,1 $\mathrm{nm} / \mathrm{sec}$ em células MDCK, $28 \mathrm{~nm} / \mathrm{sec}$ em células Caco-2 e 88\% de absorção intestinal humana; a molécula F apresentou 26 $\mathrm{nm} / \mathrm{sec}$ em células MDCK, $17 \mathrm{~nm} / \mathrm{sec} \mathrm{nm} / \mathrm{sec}$ em células Caco-2 e 78\% de absorção em células do intestino humano; e por fim a molécula $\mathrm{G}$ indicou valores de $26 \mathrm{~nm} / \mathrm{sec}$ em MDCK, $33 \mathrm{~nm} / \mathrm{sec}$ em células Caco-2 e 100\% de absorção intestinal humana (Tabela 6).

Para os parâmetros de distribuição (Tabela 5), 46\% das moléculas A e B conseguem se ligar às proteínas plasmáticas (PP) e 0,2 \% transpassam a barreira hematoencefálica (BHE); a molécula C ligou-se $100 \%$ às proteínas plasmáticas e 
perpassou 2,5\% a BHE; a molécula D se ligou 100\% às PP e perpassou 4,9\% da BHE; a molécula E se ligou $90 \%$ e transpassou 1,6\% a BHE; a molécula F se ligou $83 \%$ e transpassou $0,9 \%$ pela BHE e a molécula G ligou-se $100 \%$ às PP e 1,6\% transpassou a BHE. Para os parâmetros de metabolização, as moléculas A, B, C, F e G são metabolizadas de forma fraca pela CYP 3A4 e inibiram a CYP 2C9, já as moléculas D e E não são metabolizadas na fase um e D inibiu mais de uma CYP (CYP 2C9 e CYP 2C19).

As substâncias de estudo apresentaram, em sua maioria baixa permeabilidade em MDCK (Madin-Darby canine kidney; A, B e E), somente dois compostos apresentaram moderada absorção (F e G) e os outros dois apresentaram elevada absorção (C e D; Tabela 5), essas células são utilizadas para avaliar a permeabilidade in vivo e como essas moléculas se comportam por transporte passivo entre membranas (Irvine, et al., 1999). Todas as moléculas apresentaram moderada permeabilidade em células Caco-2 (Tabela 5), o que permite avaliar a capacidade de um fármaco ser absorvido comparado a um estudo in vivo ou ainda determinar o mecanismo de transporte de uma quantidade de possíveis fármacos (Gonçalves, 2010). Ambos tipos celulares são utilizadas para prever a absorção de compostos por formar junções entre as células (Balimane \& Chong, 2005) e todas as moléculas se mostraram capazes de serem absorvidas (Tabela 5), contudo as moléculas C, D, F e G tem maior facilidade, possivelmente por serem moléculas que apresentam maior lipofilicidade quando comparadas às outras três (Tabela 5). Somente as moléculas A e B apresentaram moderada absorção intestinal humana (Tabela 5), possivelmente este resultado é influenciado pelo baixo valor do $\log \mathrm{P}(0,23$ para ambas) e alto valor do TPSA (80,91 para ambas) que dificultam este processo.

As moléculas C, D, E, F e G se ligam de forma elevada às proteínas plasmáticas (100\%, 100\%, 90\% e 100\%, respectivamente), as moléculas A e B se ligam de forma moderada a fraca (46\% em ambas; Tabela 5). As proteínas plasmáticas são fundamentais no transporte de fármacos, se fortemente ligada, há a dificuldade, devido a diminuição da permeabilidade, do fármaco se difundir ou se transportar para o alvo, uma vez que é a forma livre - não ligada a PP - que tem a capacidade de se difundir pelas membranas (Ferreira, et al., 2020), este fato pode estar relacionado a lipossolubilidade e massa molecular destes compostos que têm valores mais expressivos (Tabela 5). Duas das moléculas (C e D) atravessam livremente a barreira hematoencefálica (Tabela 5) e busca-se como justificativa os valores de TPSA menores associado ao baixo peso molecular (Tabela 5), estas características facilitam a travessia de barreiras e contribuem para a distribuição para o SNC, enquanto que as outra cinco (A, B, E, F, G) perpassam de forma moderada a baixa (Tabela 5).

No que concerne ao metabolismo dos compostos, somente as moléculas D e E não utilizam as CYP na fase 1, quanto a inibição, todas as moléculas são capazes de bloquear a atividade da CYP_2C9 (Tabela 6), esta é uma isoforma que participa ativamente do metabolismo de uma variedade de anti-inflamatórios não esteroidais (AINES). Somente o composto D inibiu mais de uma isoforma (Tabela 6). A inibição de uma isoforma por um determinado composto pode modificar o metabolismo hepático de outro medicamento, gerando um possível acúmulo no organismo propiciando efeitos tóxicos (Brunton; Chabner \& Knollmann, 2012). O ideal é que uma possível molécula com atividade biológica promissora seja não indutora e não inibidora de CYP para assim não intervir no metabolismo de outros fármacos. 
Tabela 6. Predição dos aspectos farmacocinéticos dos monoterpenos isolados de D. ambrosioides.

\begin{tabular}{|c|c|c|c|c|c|c|c|}
\hline \multirow[t]{2}{*}{ Monoterpenos } & \multicolumn{3}{|c|}{ Absorção } & \multicolumn{2}{|c|}{ Distribuição } & \multicolumn{2}{|c|}{ Metabolismo } \\
\hline & $\operatorname{MDCK}(\mathrm{nm} / \mathrm{sec})$ & Caco-2 & HIA (\%) & $\mathbf{P P}(\%)$ & BHE & Fase 1 & Inibição \\
\hline $\begin{array}{c}\text { (1S,2S,3R,4S)-1-metil-4-(propan-2-il)ciclohexano- } \\
\text { 1,2,3,4-tetrol }\end{array}$ & 2 & 14 & 58 & 46 & 0,2 & $\begin{array}{c}\text { Fraco } \\
\text { CYP 3A4 }\end{array}$ & CYP 2C9 \\
\hline (1R,2S,3S,4S)-1,2,3,4-tetrahidroxi-p-metano & 2 & 14 & 58 & 46 & 0,2 & Fraco & CYP_2C9 \\
\hline$(1 \mathrm{R}, 2 \mathrm{~S})-3-\mathrm{p}-\mathrm{meten}-1,2$-diol & 373 & 18 & 88 & 100 & 2,5 & Fraco & CYP_2C9 \\
\hline$(1 \mathrm{R}, 4 \mathrm{~S})$-p- met-2-en-1-ol & 188 & 50 & 100 & 100 & 4,9 & - & $\begin{array}{l}\text { CYP_2C9 } \\
\text { CYP_2C19 }\end{array}$ \\
\hline 1,4-dihidroxip-met-2-eno & 0.1 & 28 & 88 & 90 & 1,6 & - & CYP 2C9 \\
\hline 1-metil-4 $\beta$ - isopropil-1-ciclohexeno- $4 \alpha, 5 \alpha, 6 \alpha$-triol & 26 & 17 & 78 & 83 & 0,9 & Fraco & CYP 2C9 \\
\hline Ascaridol & 26 & 33 & 100 & 100 & 1,6 & Fraco & CYP 2C9 \\
\hline
\end{tabular}

Legenda: MDCK - Madin-Darby Canine Kidney; Caco-2 - células de adenocarcinoma de cólon humano; HIA - Human intestinal absorption; PP - proteína plasmática; BHE - barreira hematoencefálica; CYP - citocromo P450. Parâmetros: MDCK e Caco-2: > $70 \mathrm{~nm} / \mathrm{sec}$ alta permeabilidade, 4-70 nm/sec média permeabilidade, < $4 \mathrm{~nm} / \mathrm{sec}$ baixa permeabilidade (Yazdanian, $e t$ al., 1998); HIA 0-20\% baixa absorção, 20-70\% moderada absorção, > 7-\% alta absorção; ligação a PP: ligado fortemente à albumina > 90\%, ligação de moderada a fraca à albumina < 90\% (Preadmet, 2021); BHE: > 2,0 atravessa livremente, 2,0-0,1 atravessa de forma moderada a fraca, < 0,1 atravessa de forma fraca ou não atravessa (Ajay, et al., 1999). Fonte: Autores. 
Na Tabela 7, observou-se nos resultados da predição biológica, que as moléculas não foram ativas contra bactérias, fungos e vírus. Apenas a molécula $\mathrm{G}$ apresentou atividade contra protozoários, e em geral a maioria das substâncias evidenciaram um efeito protetor mucomembranoso, carminativo e anti-seborreico. Somente a molécula $\mathrm{C}$ apresentou efeito anti-inflamatório e as moléculas A, B, C e F apresentaram atividade anti-eczema. A molécula F mostrou efeito antineoplásico e as moléculas $\mathrm{A}, \mathrm{B}, \mathrm{Fe} \mathrm{G}$ mostraram-se ativas para o tratamento de adenoma poliposo.

A molécula $\mathrm{F}$ foi a única a apresentar atividade antineoplásica, o que contradiz o teste de carcinogenicidade em camundongos que deu positivo. Além disso, foi observado efeito anti-tumoral em ratos Swiss infectados com células tumorais na almofada da pata esquerda (tumor sólido) ou na cavidade peritoneal (tumor ascítico) com tumores de Ehrlich e tratados via intraperitoneal com $5 \mathrm{mg} / \mathrm{kg}$ de extrato hidroalcoólico de folhas de D. ambrosioides L. (Nascimento, et al., 2006), possivelmente essa atividade se deu devido ao sinergismo das moléculas presentes no extrato, diferente da particularidade de um estudo in silico. Há estudos que também relacionam o Ascaridol com possível atividade antineoplásica (Efferth, et al., 2002) mas neste estudo in silico não apontou tal atividade.

Somente a molécula $\mathrm{C}$ apresentou atividade anti-inflamatória, foi observada a eficácia do extrato hidroalcóolico das folhas de $D$. ambrosioides no tratamento de osteoartrite induzida em ratos Wistar, por reduzir a inflamação sinovial característica de osteoartrite e as alterações comportamentais decorrentes da dor (Calado, et al., 2015).

As moléculas A, B, C, E, F e G apresentaram atividade protetora mucomembranosa, a literatura aponta que alguns compostos bioativos são responsáveis pela atividade gastroprotetora, sendo estes: flavonoides, esteroides e terpenos, catequinas e antocianidinas (Hurtado, 2014). O que corrobora com o estudo que indicam o Ascaridol como um composto isolado com atividade gastroprotetora (Zhu, et al., 2012; Wei, et al., 2014), contudo, nenhuma literatura elucidou o possível mecanismo que atribui essa propriedade à molécula. O efeito carminativo foi positivo para as moléculas A, B, D, E, F e G. Essa atividade é comumente descrita em estudos etnofarmacológicos (Jaramillo, et al., 2012), entretanto não há estudos que relatem compostos envolvidos nessa atividade.

Em relação às atividades antibacteriana, antifúngica e antiviral, as moléculas não foram ativas. Estudos anteriores, mostra que o extrato hidroalcoólico das folhas de D. ambrosioides apontou atividade inibitória contra Candida albicans (CIM de $0,25 \mathrm{mg} / \mathrm{mL}$ ), promovendo efeito anti-biofilme, os autores relacionam essa atividade aos metabólitos quercetina e kaempferol, devido estarem presentes em grande quantidade na composição química extrato testado (Zago, et al., 2019). Outros estudos avaliaram a atividade antibacteriana da espécie, no entanto, não se mostraram promissores para atividade. Em teste de difusão em ágar, o extrato hidroalcoólico das folhas de mastruz não promoveu efeito inibitório contra cepas de Staphylococcus aureus e Streptococcus mutans (Jácome, et al., 2020).

A molécula ascaridol (G) mostrou-se positiva para atividade antiprotozoária, o que corrobora com alguns estudos que mostram atividade contra Trypanosoma cruzi, Plasmodium falciparum e Leishmania amazonensis em que esse composto é promissor para os protozoários (Pollack, et al., 1990; Kiuchi, et al., 2001; Monzote, et al., 2007). 
Research, Society and Development, v. 10, n. 8, e9210816715, 2021

(CC BY 4.0) | ISSN 2525-3409 | DOI: http://dx.doi.org/10.33448/rsd-v10i8.16715

\begin{tabular}{|c|c|c|c|c|c|c|c|}
\hline ATIVIDADE & $\mathbf{A}$ & $\mathbf{B}$ & $\mathbf{C}$ & D & $\mathbf{E}$ & $\mathbf{F}$ & $\mathbf{G}$ \\
\hline Antineoplásico & - & - & - & - & - & + & - \\
\hline Tratamento de adenoma poliposo & + & + & - & - & - & + & + \\
\hline Antiprotozoário & - & - & - & - & - & - & + \\
\hline Antibacteriano & - & - & - & - & - & - & - \\
\hline Antifúngico & - & - & - & - & - & - & - \\
\hline Antiviral & - & - & - & - & - & - & - \\
\hline Anti-inflamatório & - & - & + & - & - & - & - \\
\hline Anti-eczema & + & + & + & - & - & + & - \\
\hline Antisseborreico & + & + & + & + & + & + & - \\
\hline Protetor mucomembranoso & + & + & + & - & + & + & + \\
\hline Carminativo & + & + & + & + & + & + & + \\
\hline
\end{tabular}

Legenda: (A) (1S,2S,3R,4S)-1-metil-4-(propan-2-il)ciclohexano-1,2,3,4-tetrol; (B) (1R,2S,3S,4S)- 1,2,3 , 4-tetrahidroxi-p-metano; (C) (1R,2S)-3-p-meten-1,2-diol; (D) (1R,4S)-p-met-2-en-1ol;(E)1,4-dihidroxip-met-2-eno;(F)1-metil-4 $\beta$-isopropil-1-ciclohexeno $4 \alpha, 5 \alpha, 6 \alpha$-triol;(G)Ascaridol. 
$\mathrm{Na}$ avaliação da toxicidade em organismos aquáticos, todas as moléculas apresentaram toxicidade para algas; nenhuma foi tóxica para crustáceos Daphnia, esses testes permitem avaliar parâmetros de intoxicação aguda e subcrônica. As moléculas C, D e G apresentaram-se muito tóxica aos peixes Medeka, e as moléculas E e F mostraram-se tóxicas. Para a espécie de peixe Minnow as substâncias A e B foram prejudiciais, os compostos C, D, E e G foram muito tóxicas, enquanto que A, B e F foram somente tóxicas. Em relação ao teste de mutagenicidade, as moléculas C, D, E, F e G se revelaram mutagênicas e as A e B não mutagênicas; para o teste de carcinogenicidade, as moléculas A, B, C, D, E, F e G não foram carcinogênicas para ratos, contudo as substâncias F e G mostram-se carcinogênicas para camundongos (Tabela 8).

As moléculas C, D e G se exibiram muito tóxicas para ambas espécies de peixes do estudo $(<1 \mathrm{mg} / \mathrm{L})$, o que indica que estas são capazes de causar intoxicações aguda e crônica, possivelmente estes resultados compartilham com o fato de estes compostos aparentarem ter maior lipossolubilidade e massa molar, permanecendo por mais tempo no organismo e facilitando as intoxicações quando comparadas às moléculas A e B, que exibiram padrão prejudicial para peixes Medeka e tóxico para Minnow (Tabela 8), essas por sua vez apresentam padrões fisico-químicos que contribuem para uma excreção mais rápida do organismo.

$\mathrm{Na}$ análise de mutagenicidade os isolados C, D, E, F e G se apresentaram mutagênicos, exceto A e B (Tabela 8) que tiveram resultado negativo. $\mathrm{E}$ em teste de carcinogenicidade, os compostos $\mathrm{F}$ e $\mathrm{G}$ não se apresentaram carcinogênico para ratos e carcinogênico para camundongos. Não foram encontrados estudos que comprovem o potencial mutagênico desta espécie. Em outro estudo, o extrato aquoso de D. ambrosioides causou tumor local em 58 ratos NIH Black, sugerindo que o extrato aquoso da planta pode ser carcinogênico (Kapadia, et al., 1978).

Tabela 8. Predição de toxicidade dos monoterpenos de D. ambrosioides.

\begin{tabular}{cccccccc}
\hline Monoterpenos & \multirow{2}{*}{ Algae } & Daphania & \multicolumn{2}{c}{ Peixes } & Mutagenicidade & Carcinogenicidade \\
\cline { 3 - 7 } & & & Medeka & Minnow & Ames & Rato & Camundongo \\
\hline A & 0,06 & 3,49 & 11,91 & 7,03 & NM & - \\
\hline B & 0,06 & 3,49 & 11,91 & 7,03 & NM & - & - \\
\hline C & 0,04 & 0,79 & 0,65 & 0,53 & Mutagênico & - & - \\
\hline D & 0,03 & 0,43 & 0,20 & 0,17 & Mutagênico & - & - \\
\hline E & 0,05 & 1,01 & 1,05 & 0,74 & Mutagênico & - & - \\
\hline F & 0,05 & 2,15 & 4,57 & 1,69 & Mutagênico & - & + \\
\hline G & 0,03 & 0,27 & 0,07 & 0,04 & Mutagênico & - & + \\
\hline
\end{tabular}

Parâmetros: NM: não mutagênico; Teste Algas: < 1mg/L tóxico, > 1mg/L não tóxico (Costa, et al., 2008); Teste Daphnia: < 0,22 $\mu$ g/mL tóxico, > 0,22 $\mu \mathrm{g} / \mathrm{mL}$ não tóxico (Guilhermino, et al., 2000); Teste em peixes Medeka e Minnow: < 1mg/L muito tóxico, 1-10 mg/L tóxico, $10-100 \mathrm{mg} / \mathrm{L}$ prejudicial e > 100mg/L não tóxico (Zuncker, 1985). Fonte: Autores.

\section{Conclusão}

Em relação aos aspectos farmacognósticos, a droga vegetal cumpre os critérios de qualidade exigidos pela $6^{\circ}$ edição da Farmacopéia Brasileira. Na prospecção fitoquímica, observou-se no extrato etanólico da folha e do caule a presença de saponinas, heterosídeos flavônicos e triterpenos e esteroides. A partir da prospecção fitoquímica e do estudo de literatura, selecionou-se sete monoterpenos para estudos in sílico por apresentarem atividade biológica, nas análises físico-químicas, todos as moléculas seguiram a regra dos 5 de Lipinski e apresentaram padrões farmacocinéticos satisfatórios, as moléculas C, F e G mostraram-se promissoras para atividade anti-inflamatória, antineoplásica e antiprotozoária, respectivamente. Contudo, são necessários estudos computacionais mais aprofundados relacionados à suas estruturas químicas, haja vista que algumas das moléculas mostraram ser carcinogênicas para camundongos, visando identificar o porquê dos efeitos indesejados e como poderia ser melhorada sem afetar as ações terapêuticas, além disso, as moléculas evidenciaram um efeito protetor 
mucomembranoso, carminativo e anti-seborreico. Entretanto, estudos pré-clínicos e clínicos são primordiais para elucidação das atividades e dos mecanismos de ação dessas substâncias isoladas.

\section{Referências}

Ahmed, A (2000). Highly Oxygenated Monoterpenes from Chenopodium ambrosioides. Journal of Natural Products, 63(7), 989-991. 10.1021/np990376u.

Ajay, A., Bermis, G. W. \& Murkco, M.A. (1999). Designing libraries with CNS activity. JMed Chem.,42(24), 4942-4951. 10.1021/jm990017w

Amarante, C. B. (2010). Estudo químico, farmacognóstico, atividade biológica e farmacológica de Montrichardia linifera (Arruda) Schott. Tese, Universidade Federal do Pará, Instituto de Ciências Exatas e Naturais, Belém, Pará, Brasil.

Ames, B. N., Mccann, J. \& Yamasaki, E. (1975). Methods for detecting carcinogens and mutagens with the salmonella/mammalian-microsome mutagenicity test*. Mutation Research. 31, 347-364. 10.1016/0165-1161(65)90046-1.

Arisawa, M., Minabe, N., Saeki, R., Takakuwa, T. \& Nakaoki, T. (1971). Studies on unutilized resources. V. The Components of the flavonoids in Chenopodium genus plants. 1. Flavonoids of Chenopodium ambrosioides L. Yakugaku Zasshi. 91(5),522-524. 10.1248/yakushi1947.91.5_522.

Balimane, P. V. \& Chong, S. (2005). Cell cultures-based models for intestinal permeability: a critique. Drug Discovery Today. 10 (5), 335-343. 10.1016/ S1359-6446 (04) 03354-9.

Barbosa, W. L. R., Pinto, L. N., Silva, W. B., Fernandes, J. G. S. \& Soler, O. (2014). Etnofarmácia do município de Igarapé-Miri - PA. In: Etnofarmácia Fitoterapia popular e ciência farmacêutica. NUMA/UFPA, 73(3), 300-11.

Blumenthal, K. D. \& Garrison, C. J. Pharmacodynamic: drug action mecanisms. In: Brunton, L.L.; Chabner, B.A.; Knollmann, B.C. Goodman \& Gilman's phamacological basis of therapeutics. $12^{\circ}$ ed. New York: ARTMED. 2012.

Brandão, D. L. N (2012). Portulaca pilosa L e Geissospermum velosii. Estudos botânicos, farmacognósticos, fitoquímicos e atividades biológicas. Dissertação de mestrado em ciências farmacêuticas. Universidade Federal do Pará, Pará, Brasil.

Calado, G. P., Lopes, A. J., Júnior, L. M. C., Lima, F. C. A, Silva, L., Pereira, W., Amaral, F. M. M., Garcia, J. B. S., Cartágenes, M. S. S. \& Nascimento, F. R. F. (2015). Chenopodium ambrosioides L. Reduces Synovial Inflammation and Pain in Experimental Osteoarthritis. PLOS ONE, 10(11), 141-886. 10.1371/ journal.pone.0141886.

Calzada, F., Arista, R. \& Pérez, H. (2010). Effect of plants used in Mexico to treat gastrointestinal disorders on charcoal-gum acacia-induced hyperperistalsis in rats. Journal of Ethnopharmacology, 128(1), 49-51. 10.1016/ j.jep.2009.12.022.

Carrillo-López, L. M, Soto-Hernández, R., Zavaleta-Mancera, H. \& Vilchis-Néstor, A. R. (2016). Study of the Performance of the Organic Extracts of Chenopodium ambrosioides for Ag Nanoparticle Synthesis. Journal of Nanomaterials, 2016, 1-13. 10.1155/ 2016/ 4714162.

Cartaxo, S. L., Sousa, M. M. A. \& Albuquerque, U. P. (2010). Medicinal plants with bioprospecting potential used in semi-arid northeastern Brazil. Journal of Ethnopharmacology, 131, 326-342. 10.1016/ j.jep.2010.07.003.

Costa, C. R., Olivi, P., Botta, C. M. R. \& Espindola, E. L. G. (2008). A toxicidade em ambientes aquáticos: discussão e métodos de avaliação. Química nova. 3(7), 1820-1830.

Costa, A. F (2001). Farmacognosia. (3 ed.). Lisboa: Fundação Calouste Gulbenkian.

Dembitsky, V., Shkrob, I. \& Lumir, L. O. (2008). Ascaridole and related peroxides from the genus chenopodium. Biomedical Papers. 152 (2), 209-215. $10.5507 /$ bp.2008.032.

Di, L. \& Kerns, E. H. (2015). Drug-like properties: concepts, structure design and methods from ADME to toxicity optimization. (2a ed.) Amsterdam: Academic press.

Efferth, T., Olbrich, A., Sauerbreya A., Ross, D. D., Gebhart, E. \& Neugebauer, M. (2002). Activity of ascaridol from the anthelmintic herb Chenopodium anthelminticum L. against sensitive and multidrug-resistant tumor cells. Anticancer Research. 22(6C),4221-4224.

Farmacopeia Brasileira Agência Nacional de Vigilância Sanitária - Anvisa (2019). https://www.gov.br/anvisa/pt-br/assuntos/farmacopeia/farmacopeiabrasileira/arquivos/7985json-file-1

Filimonov, D. A., Poroikov, V. V., Karaicheva, E. I., Kazarian, R. K., Budunova, A. P. \& Mikhailovskii, E. M. (1995). The computerized prediction of the spectrum of biological activity of chemical compounds by their structural formula: the PASS system. Prediction of activity spectra for substances. Eksp Klin Farlakol. 58(2), 56-62.

Fontenele, R. (2017). Estudos etnodirigidos, obtenção de fitoterápico e controle de qualidade: um estudo de caso com Chenopodium ambrosioides L. Dissertação de mestrado em ciências farmacêuticas. Universidade Federal do Piauí, Piauí, Brasil.

Garcia, D., Domingues, M. V., \& Rodrigues, E. (2010). Ethnopharmacological survey among migrants living in the Southeast Atlantic Forest of Diadema, Sao Paulo, Brazil. Journal of Ethnobiology and Ethnomedicine, 6(1), 29. 10.1186/1746-4269-6-29.

Ghareeb, M. A., Saad, A. M., Abdou, A. M., Refahy, L. A. \& Ahmed, W. S. (2016). A New Kaempferol Glycoside with Antioxidant Activity from Chenopodium ambrosioides Growing in Egypt. ResearchGate; Oriental Scientific Publishing Company. 32(6), 3053-3061. http://dx.doi.org/10.13005/ojc/320626. 
Gonçalves, J. E. (2010). Padronização das condições para cultura de células Caco-2 visando à obtenção de membranas viáveis ao estudo da permeabilidade in vitro da rifampicina. Tese de Doutorado, São Paulo: FCF-USP. Recuperado Https://teses.usp.br/teses/disponiveis/9/9139/tde-31012011-162346/pt-br.php.

Grassi, L. T (2011). Chenopodium ambrosioides L. Erva de santa maria (amaranthaceae): estudo do potencial anti-inflamatório, antinociceptivo e cicatrizante. Dissertação de mestrado. Universidade do Vale do Itajaí, Itajaí, Brasil.

Guilhermino, L., Diamantino, T., Silva, M. C. \& Soares, A. M. V. M. (2000). Acute toxicity test with Daphnia magna: An alternative to mammails in the Prescreening of Chemical Toxicity? Ecotoxicology and Environmental Safety. 46(3), 357-362. 10.1006/ eesa.2000.1916.

Hallal, A., Markouk, M., Bekkouche, K., \& Larhsini, M. (2010). Evaluation of the analgesic and antipyretic activities of Chenopodium ambrosioides L. Sian Journal of Experimental Biological Sciences. 1 (1),189-192.

Hurtado P (2014). Evaluación de la actividad gastroprotectora del extracto hidroalcohólico de las hojas de Juglans neotropica Diels "Nogal peruano. Tese de doutorado. Universidad Nacional Mayor de San Marcos, Lima, Peru.

Irvine, J. D., Takahashi, L, Lockhart, K., Cheong, J., Tolan, J. W., Selick, H. E. \& Grove, J. R. (1999). MDCK (Madin-Darby canine kidney) cells: Atool for membrane permeability Screening. J Pharm Sci, 88(1), 28-33. 10.1021/ js9803205.

Jácome, E. V. et al (2020). Atividade antibacteriana de extratos hidroalcoólicos de Chenopodium ambrosioides (mastruz) e Crescentia cujete (coité) em Streptococcus mutans e Staphylococcus aureus. Revista Eletrônica Acervo Saúde, 12(10),47-87. 10.25248/ reas.e4787.2020.

Jain, N., Alam, M. S., Kamil, M., Ilyas, M., Niwa, M. \& Sakae, A. (1990). Two flavonol glycosides from Chenopodium ambrosioides. Phytochemistry. 29(12),3988-91. 10.1016/ 0031-9422(90)85389-W.

Jaramillo, B. E. C., Duarte, E. R. \& Delgado, W. (2012). Bioatividad del aceite esencial de Chenopodium ambrosioides colombiano. Jornal Cubano de Plantas Medicinais, 17(1), 54-64.

Lima Júnior, J. A. C., Costa, G. C., Reis, A. S., Bezerra, J. L., Patrício, F. J. B., Silva, L. A., Amaral, F. M. M. \& Nascimento, F. R. F. (2014). Inibição da infecção invitro de macrófagos por Leishmania amazonenses por extrato e frações de Chenopodium ambrosioides L.. Health Science Journal. 16(1), 46-53.

Kapadia, G. J., Chung, E. B., Gosh, B., Shukia, Y. N., Basak, S. P., Monrton, J. F. \& Pradhan, S. N. (1978). Carcinogenicity of Some Folk Medicinal Herbs in Rats. Journal of the national cancer institute, 60(3), 683-686. 10.1093/jnci/60.3.683.

Kiuchi, F., Itano, Y., Uchiyama, N., Honda, G., TsubouchiI, A., Nakajima-Shimada, J. \& Aoki, T. (2002). Monoterpene hydroperoxides with trypanocidal activity from Chenoopodium ambrosioides. Journal of Natural Products. 65(4), 509-512. 10.1021/np10445g.

Kubinyi, H. (1977). Quantitative structure-activity relations. 7. The bilinear model, a new model for nonlinear dependence of biological activity on hydrophobic character. Journal of Medicinal Chemistry. 20(5), 625-629. 10.1021/jm00215a002.

Lachman, L., Lieberman, H. A. \& Kanig, J. L (2001). Teoria e Prática na Indústria Farmacêutica. Fundação Calouste Gulbenkian.

Lipinski, C. A. (2005). Lead-and drug-like compounds: the rule of five revolution. Drug Discovery today: Technologies. 1(4), 337-341. 10.1016/j.ddtec.2004.11.007.

Lipinski, C. A., Lombardo, F., Dominy, B. W. \& Feeney, P. J. (1997). Abordagens experimentais e computacionais para estimar a solubilidade e permeabilidade em configurações de desenvolvimento e descoberta de drogas. Advanced Drug Delivery Reviews. 23, 3-25.

Lorenzi, H. \& Matos, F. J. A. (2002). Plantas medicinais no Brasil: nativas e exóticas. Plantarum.

MOLINSPIRATION. Prediction of chemical properties.

Montanheiro, M. N. S. (1990). Determinação da densidade de sólidos e líquidos pelo príncipio de Arquemedes. Caderno catarinense de ensino de Física, 7(2), $120-123$.

Monzote, L., Margarida, A., Lizama, R. S. \& Payrol, J. A. (2007). Activity, toxocity and analisys of resistance of essential oil fro Chenopodium ambrosioides after intraperitoneal, oral and intralesional administration in BALB/c mice infected with Leishmania amazonensis: a preliminary study. Biomed Pharmacoterapy, 61 (2-3), 148-53. 10.1016/j.biopha.2006.12.001.

Monzote, L., Stamberg, W., Staniek, K. \& Gille, L. (2009). Toxic effects of carvacrol, caryophyllene oxide and ascaridole from essential oil of Chenopodium ambrosioides on mitochondria. Toxicology and Applied Toxicology. 240(3), 337-347. 10.1016/j.taap.2009.08.001Epub.

Morais, S. M., Dantas, J. D. P., Silva, A. R. A., Magalhães, E. F. (2005). Plantas medicinais usadas pelos índios Tapebas do Ceará. Revista Brasileira de Farmacognosia, 15(2), 169-177. 10.1590/S0102-695X2005000200017.

Mosyakin, S. L. \& Clemants, S. E. (2008). Futher transfers og glandular-pubescent species from Chenopodium subg. Ambrosia to dysphania (Chenopodiaceae). The botanical research institute of Texas, 2(1), 425- 431.

Nascimento, F. R. F., Cruz, G. V. B., Pereira, P. V. S., Maciel, M. C. G., Silva, L. A., Azevedo, A. P. S., Barroqueiro, E. S. B. \& Guerra, R. N. M. (2006). Ascitic and solid Ehrlich tumor inhibition by Chenopodium ambrosioides L. treatment. Life Sciences 78(22), 2650-2653. 10.1016/j.lfs.2005.10.006.

Pandiangan, D., Lamlean, P. Y., Maningkas, P. F. \& Unitly, A. J. A. (2020). Antioxidant and Anticancer Activity Tests of "Pasote" Leaf Water Extracts (Dysphania ambrosioides L.). ResearchGate. 1463, 1-9. 10.1088/1742-6596/1463/1/012020.

PASS Online. Prediction of biology activity 
Research, Society and Development, v. 10, n. 8, e9210816715, 2021

(CC BY 4.0) | ISSN 2525-3409 | DOI: http://dx.doi.org/10.33448/rsd-v10i8.16715

PREADMET. Admet prediction.

Pereira, D G (2007). Importância do metabolismo no planejamento de fármacos. Química Nova,30(1),171-177.

Pollack, Y., Segal, R. \& Gollenser, J. (1990). The effect of Ascaridole on the in vitro development of Plasmodium falciparum. Parasitology research, 76(7), 570-572. 10.1007/BF00932563.

Ramos, U. F., Soledade, S. C. \& Baptista, E. R. (2011). Utilização de plantas medicinais pela comunidade atendida no Programa Saúde da Família da Pirajá, Belém, PA. Infarma. 24(5-6), 10-18.

Simões, C. M. O., Mentz, L. E., Schenkel, E. P., Irgang, B. E. \& Stehmann, J. R. (2010). Plantas da Medicina Popular do Rio Grande do Sul, Eduni-Sul: Porto Alegre, Brasil. Revista Brasileira de Farmacognosia. 12(2),51-62. 10.1590/S0102-695X200200200001

Silva, D. R., Ferreira, S. A. M., Silva, T. S., Sette-de-Sousa, P. H. \& Silva, A. C. B. (2018). Atividade antimicrobiana do extrato de Chenopodium ambrosioides e Ruta graveolens sobre Streptococcus mutans. Archive of health investigation. 7(4),120-122. 10.21270/archi.v7i4.2821.

Shah, H. \& Khan, A. A. (2017). Phytochemical characterisation of an important medicinal plant, Chenopodium ambrosioides Linn. Natural Product Research. 31(19), 2321-2324. 10.1080/14786419.2017.1299722.

Shu-Qun, H., Yan-Hong, L., Xiang-Zhong, H., Rong, L., Huai, L., Kai, T. \& Rong-Sheng, R. (2017), L. Polyol monoterpenes isolated from Chenopodium ambrosioides. Natural product research. Austrália, 31(21), 2467-2472. 10.1080/14796419.2017.1314278.

Trindade, G. D., Filho, A. L. M. M., Rodrigues, J. S., Ferreira, D. C. L. \& De Araújo, K. S. (2021). Efeitos de extrato em gel de chenopodium ambrosioides L. (mastruz) no tratamento de lesões ósseas de ratas osteoporóticas. Revista Eletrônica Acervo Odontológico. 3(e6260),1-8. DOI:10.25248/reaodonto.e6260.202.

Valério, E. (2014). Avaliação da atividade dos extratos hidroetanólico de Chenopodium ambrosioides L. e de Eucalyptus alba Reinw ex Blume, frente a cepas de Mycobacterium sp. Dissertação de mestrado. Universidade Federal do Pará, Belém, Pará, Brasil.

Veber, D. F., Johnson, S. R., Cheng, H. Y., Smith, B. R., Ward, K. W. \& Kopple, K. D. (2002). Molecular Properties That Influence the Oral Bioavailability of Drug Candidates. Journal of Medicinal Chemistry. 45(12), 2615-2623. 10.1021/jm020017n.

Wagner, H., Bladt, S. \& Zgainski, E. M. (1984). Plant drug analysis. Springer.

Yazdanian, M., Glynn, S. L., Wright, J. L., Hawi, A. (1998). Correlating partitioning and Caco-2 Cell Permeability of Structurally Diverse Small Molecular Weight Compounds. Pharmaceutical Research. 15(9), 1490-1494.

Yee S. (1997). In vitro permeability across Caco-2 cells (colonic) can predictin vivo (small intestinal) absorption in man-fact or myth. Pharm Res.. 14(6), 763766. 10.1023/a:1012102522787.

Zago, P. M. W., Branco, S. J. S. C., Fecury, L. A. B., Carvalho, L. T., Rocha, C. Q., Madeira, P. L. B., Sousa, E. M., Siqueira, F. S. F., Paschoal, M. A. B., Diniz, R. S. \& Gonçalves, L. M. (2019). Anti-biofilm Action of Chenopodium ambrosioides Extract, Cytotoxic Potential and Effects on Acrylic Denture Surface. Frontiers in Microbiology. 10, 1-9. 10.3389/fmicb.2019.01724.

Zhu, W. X., Zhao, K., Chu, S. S. \& Liu, Z. L. (2012). Evaluation of Essential Oil and its Three Main Active Ingredients of Chinese Chenopodium ambrosioides (Family: Chenopodiaceae) against Blattella germanica. Journal of Arthropod-Borne Diseases. 6(2), 90-97.

Zorzeto, T. Q., Dechen, S. C. F., Abreu, M. F. \& Fernandes Júnior, F. (2014). Caracterização física de substratos para plantas. Bragantia, 73(3), 300-311. 10.1590/1678-4499.0086.

Zucker, E. (1985). Hazard Evaluation Division Standard Evaluation Procedure: Acute toxicity test for freshwater Fish. USEPA. Recuperado de https://ntrl.ntis.gov/NTRL/dashboard/searchResults/titleDetail/PB86129277.xhtml 